Pontifícia Universidade Católica $_{\text {a }}$

DO RIO DE JANEIRO

Cristina Portugal

Design em Situações de Ensino-aprendizagem.

Um diálogo interdisciplinar

Tese de Doutorado

Tese apresentada ao Programa de Pós-graduação em Design da PUC-Rio como requisito parcial para obtenção do título de Doutor em Design.

Orientador: Rita Maria de Souza Couto

Co-orientador: Juan Carlos Arañó Gisbert

Rio de Janeiro, dezembro de 2009 


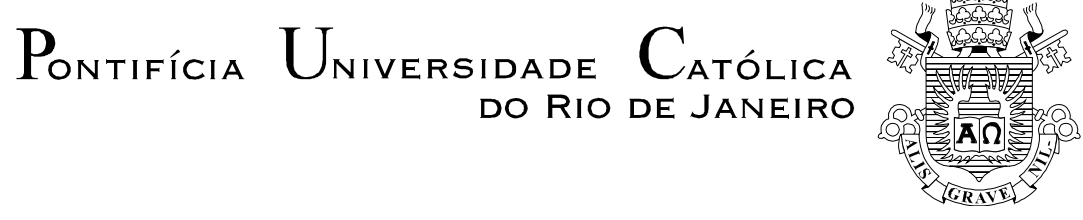

Cristina Portugal

Design em Situações de Ensino-aprendizagem Um diálogo interdisciplinar

Tese apresentada como requisito parcial para obtenção do grau de Doutor pelo Programa de Pós-graduação em Design da PUC-Rio. Aprovada pela Comissão examinadora abaixo assinada.

\author{
Profa. Rita Maria de Souza Couro \\ Orientadora \\ PUC-Rio \\ Profa. Flávia Nizia da Fonseca Ribeiro \\ UNIRIO
}

Prof. Daniel Wyllie Lacerda Rodrigues UFRJ
Profa. Jackeline Lima Farbiarz PUC-Rio

Profa. Denise Berruezo Portinari

PUC-Rio

Prof. Paulo Fernando Carneiro de Andrade Coordenador Setorial do Centro de Tecnologia e Ciências Humanas - PUC-Rio

Rio de Janeiro, 14 de dezembro de 2009 
Todos os direitos reservados. É proibida a reprodução total oi parcial do trabalho sem a autorização da universidade, da autora e do orientador.

\section{Cristina Portugal}

Doutor em Design, Mestre em Design e Bacharel em Desenho Industrial com habilitação de Comunicação Visual pela Pontifícia Universidade Católica do Rio de Janeiro - PUC-Rio. É pesquisadora do Laboratório Interdisciplinar de Design/Educação -LIDE do Departamento de Artes \& Design da PUC-Rio.

Ficha catalográfica

Portugal, Cristina

Design em situações de ensino-aprendizagem : um diálogo interdisciplinar / Cristina Portugal ; orientadora: Rita Maria de Souza Couto ; coorientador: Juan Carlos Arañó Gisbert. - 2009 206 f. : il. ; $30 \mathrm{~cm}$

Tese (Doutorado em Design)-Pontifícia Universidade Católica do Rio de Janeiro, Rio de Janeiro, 2009.

Inclui bibliografia

1. Artes - Teses. 2. Design em situações de ensino-aprendizagem. 3. Interdisciplinaridade. 4. Cultura visual. 5. Jogo. 6. Multi-trilhas. I. Couto, Rita Maria de Souza. II. Gisbert, Juan Carlos Arañó. III. Pontifícia Universidade Católica do Rio de Janeiro. Departamento de Artes \& Design. IV. Título. 


\section{Agradecimentos}

À professora e orientadora Rita Couto pela amizade, generosidade, dedicação, compromisso, compreensão e incentivo.

Ao professor e co-orientador Juan Carlos Arañó pela acolhida, carinho e apoio durante todo estágio sanduíche na Universidade Sevilha.

À CAPES pelo apoio financeiro em forma de bolsa no programa de Doutorado com Estágio no Exterior - PDEE realizado na Universidade de Sevilha.

À PUC-Rio pela concessão da bolsa VRAC que possibilitou a realização desta pesquisa.

Aos meus colegas do Laboratório Interdisciplinar de Design/Educação - LIDE, em especial, Flávia Nizia, Bianca Martins, Patrícia Castro e Cida Bernabó pela amizade e que de alguma maneira contribuíram para a discussão, debate e divulgação das idéias contidas neste trabalho; e também, por tornar o caminho percorrido para o desenvolvimento desta tese tão rico e divertido.

Aos professores do Departamento de Artes \& Design da PUC-Rio, em especial Denise Portinari e João Leite, que através do seu interesse, competência e dedicação muito contribuíram para esta tese.

Aos funcionários do Departamento de Artes \& Design da PUC-Rio, em especial ao Romário, por sua atenção.

Aos meus irmãos, em especial Virgínia, Denise, Valeria e Guilherme pelo carinho, por clarear minhas idéias e por estarem sempre presentes nos momentos mais difíceis.

A minha querida irmã Valeria pelo seva.

A Ana Lúcia pelo carinho, apoio e amizade.

A todos que direta ou indiretamente me ajudaram a realizar este trabalho.

Agradeço em especial aos meus queridos pais, Maria Isabel e Roberto pelo amor e apoio em todos os momentos. E, ao meu amado filho Felipe por ser especial. 


\section{Resumo}

Portugal, Cristina; Couto, Rita Maria de Souza. Design em Situações de Ensinoaprendizagem. Um diálogo interdisciplinar, Rio de Janeiro, 2009. 206p. Tese (Doutorado em Design) - Departamento de Artes \& Design, Pontifícia Universidade Católica do Rio de Janeiro.

Esta tese refere-se ao estudo, pesquisa e fundamentação teórica da linha de investigação Design em Situações de Ensino-aprendizagem, por meio de um diálogo interdisciplinar entre Design e Educação. Buscou-se através de métodos e técnicas de Design identificar de que maneira esta área de conhecimento pode participar dos processos de ensino-aprendizagem, na configuração de materiais pedagógicos, bem como potencializar o processo de aquisição de conhecimento. A pesquisa teve por fio condutor o Multi-Trilhas, objeto de estudo desta tese, que é um jogo para auxiliar a aquisição de segunda língua por crianças surdas. Este jogo foi desenvolvido à luz de métodos e técnicas de Design, no âmbito do Laboratório Interdisciplinar de Design/Educação, da Pontifícia Universidade Católica do Rio de Janeiro, em parceria com o Instituto Nacional de Educação de Surdos no Rio de Janeiro. Por meio da articulação de idéias de autores que fundamentam esta pesquisa, foram trabalhadas questões relacionadas a novas tecnologias, cultura visual, cultura em geral, imagem, linguagem e construção de significados, aspectos considerados relevantes para campo do Design de forma geral e para subsidiar a conceituação da linha de investigação Design em Situações de Ensino-aprendizagem, bem como foram realizados estudos sobre pesquisas de mestrado e de doutorado que tratam do Design na Educação, enfocando, entre outros aspectos, questões de ensino-aprendizagem; jogo, linguagem e práticas pedagógicas visuais. A presente investigação estabelece uma proposta de modelo conceitual de ação pedagógica e uma metodologia para a execução de projetos no âmbito da linha de pesquisa Design em Situações de Ensino-aprendizagem. A pesquisa visa, também, contribuir para o planejamento de materiais educacionais que considerem as especificidades educacionais, seu diálogo e trânsito com a realidade circundante, objetivando o desenvolvimento de processos de ensino-aprendizagem mais produtivos e interativos.

\section{Palavras-chave}

Design em Situações de Ensino-aprendizagem; Design/Educação; Interdisciplinaridade; Cultura Visual; Jogo; Multi-Trilhas. 


\section{Abstract}

Portugal, Cristina; Couto, Rita Maria de Souza (Advisor). Design in Situations of Teaching and Learning. An interdisciplinary dialogue, Rio de Janeiro, 2009. 206p. Dsc. Thesis - Departamento de Artes \& Design, Pontifícia Universidade Católica do Rio de Janeiro.

This thesis refers to the study, research and theoretical line of research Design in Situations of Teaching and Learning through an interdisciplinary dialogue between Design and Education. Through methods and techniques of design it was sought to identify how this area of knowledge can participate in the processes of teaching and learning at the setting of learning materials and enhance the process of acquiring knowledge. The study was guided by the Multi-Tracks, subject of this thesis, which is a game to help the acquisition of a second language by deaf children. This game was developed in the light of methods and techniques of Design, under the Interdisciplinary Laboratory for Design / Education - LIDE, Catholic University of Rio de Janeiro - PUC-Rio, in partnership with the National Institute of Education for the Deaf in Rio de Janeiro - INES / RJ. Through the articulation of ideas of authors who support this research we worked issues related to new technologies, visual culture, culture in general, image, language and meaning making, relevant aspects for the design field in general and to subsidize conceptualization of the line of research Design in Situations of Teaching and Learning, and studies were performed on research at the level of Mastership and Doctorate dealing with Design in Education, focusing among other things, issues of teaching and learning, games, language and visual pedagogic techniques. This research provides a proposal for a conceptual model of pedagogical action and a methodology for the implementation of projects under the line of research Design in Situations of Teaching and Learning. The research also aims to contribute to the planning of educational materials that consider the specific educational traits and their dialogue with the surrounding reality, aiming at developing methods of teaching and learning that are more productive and interactive.

\section{Keywords}

Design in Situations of Teaching and Learning; Design/Education; Interdisciplinarity; Visual Culture; Game; Multi-Tracks. 


\section{Resumen}

Portugal, Cristina; Couto, Rita Maria de Souza. Diseño en Situaciones de Enseñanza y de Aprendizaje, por medio de un diálogo entre Diseño y Educación, Río de Janeiro, 2009. 206p. Tesis (Doctorado en Diseño) - Departamento de Artes \& Design, Pontifícia Universidade Católica do Rio de Janeiro.

Esta tesis se refere al estudio, investigación y construcción del marco teórico de la línea de investigación Diseño en Situaciones de Enseñanza y de Aprendizaje, por medio de un diálogo entre Diseño y Educación. Se buscó a través de los métodos y de las técnicas de Diseño identificar de qué forma esta área de conocimiento puede participar de los procesos de Enseñanza y de Aprendizaje en la configuración de materiales pedagógicos, bien como intensificar el proceso de adquisición de conocimiento. La investigación tuvo por hilo conductor el Multi-Trilhas, objeto de estudio de esta tesis, que es un juego hecho para ayudar la adquisición de la segunda lengua por niños sordos. Este juego fue desarrollado con base en métodos y técnicas de Diseño, en el ámbito del Laboratorio Interdisciplinar de Diseño/Educación de la Pontificia Universidad Católica de Río de Janeiro, Brasil, en sociedad con el Instituto Nacional de Educación de Sordos de Río de Janeiro. Por medio de la articulación de ideas de autores que fundamentan esta investigación, fueron trabajadas cuestiones relacionadas a nuevas tecnologías, cultura visual, cultura en general, imagen, lenguaje y construcción de significados, aspectos considerados relevantes para el campo del Diseño de forma general y para subsidiar la conceptuación de la línea de investigación Diseño en Situaciones de Enseñanza y de Aprendizaje, bien como fueron realizados estudios sobre investigaciones de maestro y de doctorado que tratan del Diseño en la educación, enfocando, entre otros aspectos, cuestiones de Enseñanza y de Aprendizaje; Juego, lenguaje y prácticas pedagógicas visuales. La presente investigación establece una propuesta de modelo conceptual de acción pedagógica e una metodología para la ejecución de proyectos en el ámbito de la línea de investigación Diseño en Situaciones de Enseñanza y de Aprendizaje. La investigación visa, también contribuir para la planificación de materiales educacionales que consideren las especificaciones educacionales, su dialogo y tránsito con la realidad del ambiente, objetivando el desarrollo de procesos de enseñanza y de aprendizaje más productivos e interactivos.

\section{Palabras llaves}

Diseño en Situaciones de Enseñanza y de Aprendizaje; Diseño/Educación; Interdisciplinaridad; Cultura Visual; Juego; Multi-Trillas. 


\section{Sumário}

1. Introdução

2. Projeto Multi-Trilhas: Design em Situações de Ensino-aprendizagem aplicado

2.1. Histórico e metodologia do projeto Multi-Trilhas 22

2.2. Jogo Multi-Trilhas - versão concreta 33

2.3. Jogo Multi-Trilhas - versão multimídia 53

3. Imagem, linguagem e novas tecnologias de Informação e comunicação

3.1. Novas tecnologias sob a perspectiva do Design 80

3.2. Cultura visual e educação 91

3.3. Imagem e Linguagem como veículo de comunicação 103

3.4. Cultura e construção de significados 114

4. Design na Educação 129

4.1. Breve panorama de pesquisas que abordam o tema Design na Educação

4.2. Investigações sobre ensino-aprendizagem 144

4.3. Questões sobre jogo, linguagem e aprendizagem 163

4.4. Design em Situações de Ensino-aprendizagem 174

5. Conclusões e sugestões 188

6. Referências bibliográficas 195 


\section{Lista de Figuras}

Figura 01: Mapa conceitual das questões que fundamentam este $\quad 19$ estudo.

Figura 02: A relação das cartas e peças poligonais através das

cores.

Figura 03: Imagens do Pão de Açúcar dos cenários circulares. $\quad 35$

Figura 04: Imagens do corpo de bombeiro dos cenários circulares. $\quad 36$

Figura 05: Imagens do jardim zoológico dos cenários circulares. $\quad 36$

Figura 06: Modelos da primeira versão das cartas. 37

Figura 07: Estudos de ilustração visando identificar a melhor maneira 41 de representar graficamente movimentos e expressões corporais envolvidos nesta linguagem.

Figura 08: Estudos em desenho da palavra "claro" em LIBRAS.

Figura 09: Estudo de linguagens e técnicas em vetor da palavra "junto" em LIBRAS.

Figura 10: Estudo de linguagens e técnicas em vetor da palavra "querer" em LIBRAS.

Figura 11: Estudos da representação gráfica (traço, setas, linhas) da palavra "alto" em LIBRAS .

Figura 12: Estudos da representação gráfica (traço, setas, linhas) da palavra "claro" em LIBRAS.

Figura 13: Representação gráfica de palavra "vermelho" em LIBRAS. 43

Figura 14: Consultor Pedagógico Alex Curione - INES/RJ e

representação gráfica da palavra "apagar" em LIBRAS.

Figura 15: Aplicação das ilustrações nas cartas.

Figura 16: Estudos de mãos para representação da datilologia para a 45 carta-apoio.

Figura 17: Layout da carta.

Figura 18: Resultado final das cartas-ação: verbo - amarelo;

pronome - rosa; adjetivo - roxo; representados em LIBRAS.

Figura 19: Exemplos de cartas-ação. Substantivo - azul; artigo laranja.

Figura 20: Exemplos de figuras-bônus.

Figura 21: Exemplos de cartas-comando.

Figura 22: Exemplos de cartas-apoio número e alfabeto - vermelho. $\quad 49$

Figura 23: Pinos.

Figura 24: Versão final da logomarca.

Figura 25: Manual frente e verso.

Figura 26: Esquema de Padovani apresentando o input da 
(Padovani, 2006).

Figura 27: Fluxograma do objeto multimídia.

Figura 28: Esquema dos passos para jogar o jogo multimídia. $\quad 57$

Figura 29: Layout das telas do objeto multimídia. 58

Figura 30: Exemplo de telas iniciais do jogo virtual, apresentando $\quad 60$

Datilologia, LIBRAS e Português.

Figura 31: Esquema de construção de personagem do jogo 61

multimídia.

Figura 32: Outros personagens do jogo multimídia. 62

Figura 33: Tela Home 64

Figura 34: Tela de escolhas dos cenários. 65

Figura 35: Tela do cenário do Pão de Açúcar. 65

Figura 36: Tela atividade associação de imagem/palavra do cenário 65

Corpo de Bombeiro.

Figura 37: Tela Liga pontos do cenário Zôo. 66

Figura 38: Tela Quebra cabeça do cenário Pão de Açúcar. 66

Figura 39: Tela do dicionário. $\quad 67$

Figura 40: Tela de arte. $\quad 67$

Figura 41: Tela de congratulações. $\quad 67$

Figura 42: Tela de pontuação para todos os cenários. 68

Figura 43: Tela o jogo. $\quad 69$

Figura 44: Exemplo de telas do jogo Multi-Trilhas multimídia. $\quad 70$

Figura 45: Campo da produção cultural. Fonte: John A. Walker e 93

Sarah Chaplin (1997).

Figura 46: Esquema descritivo sobre linguagem de Twyman (1985). 107

Figura 47: A prática da EdaDE proposta por Fontoura, 2002, p. 280.

Figura 48: Modelo Conceitual para análise de projetos no âmbito da 159

linha de pesquisa Design em Situações de Ensino-aprendizagem

utilizando o Projeto Multi-Trilhas, proposto por Portugal (2009).

Figura 49: Mapa mental para atividade de design de Fontoura, 2002: 177 p. 188.

Figura 50: Proposta de metodologia para projetos de Design em

Situações de Ensino-aprendizagem. 


\section{Lista de tabelas}

Tabela 01: Etapas do processo de pesquisa 18

Tabela 02: Esquema de jogabilidade do jogo Multi-Trilhas concreto. $\quad 51$

Tabela 03: Esquema de jogabilidade do jogo Multi-Trilhas mutimídia. $\quad 68$

Tabela 04: Dissertações de mestrado $\quad 141$

Tabela 05: Teses de doutorado. 143

Tabela 06: Mapa esquemático da investigação sobre o ensino de 151

Shulman 1986, p. 22.

Tabela 07: Modelo conceitual e de ação pedagógica (Shulman 2008). 157

Tabela 08: Modelo conceitual para definição de projetos no âmbito da 159 linha de pesquisa Design em Situações de Ensino-aprendizagem utilizando o Projeto Multi-Trilhas como exemplo.

Tabela 09: Bases da metodologia utilizada no desenvolvimento do 177 projeto Multi-Trilhas.

Tabela 10: Proposta de metodologia de Design em Situações de Ensino- 185 aprendizagem. 